\title{
Derivation of Predicted Pressure Drop Equation on Granular Particle Plug Transportation in Horizontal Pipe"
}

\author{
$\mathrm{Kenji} \mathrm{KOFU}^{* *}$, Mitsuaki OCHI ${ }^{* *}$ and Masahiro TAKEI** \\ ** Department of Mechanical Engineering, College of Science \& Technology, Nihon University \\ 1-8-14 Kanda-Surugadai, Chiyoda-ku, Tokyo, 101-8308, Japan \\ E-mail: kofu@mech.cst.nihon-u.ac.jp
}

\begin{abstract}
In a plug transportation that is one of gas-solid two-phase flows, the prediction equations on particle velocity within a plug and pressure drop in a horizontal pipe have been formulated. The agreement between values calculated by these equations and experiments in which solid-air mass flow rate, pipe diameter, kinds of particle were changed was obtained. The difference between them is almost within $10 \%$. In order to confirm the validity of supposition to derive these equations, particle velocity distribution within a plug in the directions of flow and radius, and particle velocity transformation from a stationary bed to a plug have been analyzed by high speed camera and PIV. The results show that there is no particle velocity distribution in a plug, and particles are accelerated uniformly in extra part of a plug. Namely, particles in a plug are fixed relative to each other and so they all move with the same velocity.
\end{abstract}

Key words: Pressure Drop, Particle Velocity, Pipe Line, Gas-Solid Two-Phase Flow, PIV

\section{Introduction}

Pneumatic transportation, which is one form of particulate transportation, has many advantages over container transportation, belt conveyors, and other types of mechanical transportation. These merits include a high-speed, sanitary, and inexpensive transportation process. Pneumatic transportation can be broadly characterized as dilute-phase high-speed transportation and dense-phase low-speed transportation. Dense-phase low-speed transportation, called plug transportation, is often adopted from the viewpoint of pipe wall wear and particle breakage prevention, as well as energy savings.

In the design of a plug transportation line, it is necessary to estimate the pressure drop accurately from the viewpoint of cost and energy reduction and reliability. These factors have been studied by many researchers, and many equations to predict pressure drop have been developed ${ }^{(1)-(5)}$. However, these equations cannot be assumed to be practical because the difference between the theoretical calculation and experimental results becomes large when the transportation conditions, such as the types of particles and pipe diameter, are changed. Additionally, there is the problem of changing the coefficient in the Ergun's equation according to the particle shape ${ }^{(1)-(3)}$. For the pressure drop through a static packed bed, the author has derived a modified Ergun's equation that considers pipe diameter and particle shape ${ }^{(6)}$. The predicted results agree with experimental results within $6 \%$, and the applicability of this equation is high for many types of particles, air velocity, and pipe diameter. However, this equation requires the particle velocity and a preliminary experiment

*Received 15 Dec., 2007 (No. T2-07-0190) Japanese Original : Trans. Jpn. Soc. Mech. Eng., Vol.73 No.733, B (2007), pp.1868-1875 (Received 8 Mar, 2007) [DOI: 10.1299/jst.3.104] 
in order to apply it to plug transportation.

The purpose of this study is to derive a prediction equation for pressure drop that has a high application for various pipe diameters, types of particles and transportation conditions, and does not require a preliminary experiment. In addition, the flow conditions of the particles are measured, and the validity of the assumptions in the derivation of the prediction equation is verified.

\section{Nomenclature}

\begin{tabular}{lll}
$A$ & $:$ Pipe cross-sectional area & {$\left[\mathrm{m}^{2}\right]$} \\
$D$ & $:$ Pipe diameter & {$[\mathrm{m}]$} \\
$d_{P}$ & $:$ Particle diameter & {$[\mathrm{m}]$} \\
$F$ & $:$ Stress at the front and back end of a plug & {$[\mathrm{Pa}]$} \\
$g$ & $:$ Acceleration of gravity & {$\left[\mathrm{m} / \mathrm{s}^{2}\right]$} \\
$l_{e x}$ & $:$ Single extra plug length & {$[\mathrm{m}]$} \\
$l_{P}$ & $:$ Single plug length & {$[\mathrm{m}]$} \\
$L_{P}$ & $:$ Total plug length & {$[\mathrm{m}]$} \\
$L_{T}$ & $:$ Pipe length & {$[\mathrm{m}]$} \\
$M_{s}$ & $:$ Mass flow rate of particles & {$[\mathrm{kg} / \mathrm{s}]$} \\
$P$ & $:$ Air pressure & {$[\mathrm{Pa}]$} \\
$\Delta P$ & $:$ Pressure drop & {$[\mathrm{Pa}]$} \\
$U_{a}$ & $:$ Superficial air velocity & {$[\mathrm{m} / \mathrm{s}]$} \\
$U_{S P}$ & $:$ Slip velocity & {$[\mathrm{m} / \mathrm{s}]$} \\
$u_{s}$ & $:$ Particle velocity in the plug & {$[\mathrm{m} / \mathrm{s}]$} \\
$W_{P}$ & $:$ Plug velocity & {$[\mathrm{m} / \mathrm{s}]$} \\
$\varepsilon$ & $:$ Porosity & {$[-]$} \\
$\mu_{w}$ & $:$ Coefficient of friction & {$[-]$} \\
$\rho_{a}$ & $:$ Air density & {$\left[\mathrm{kg} / \mathrm{m}^{3}\right]$} \\
$\rho_{B}$ & $:$ Bulk density & {$\left[\mathrm{kg} / \mathrm{m}^{3}\right]$} \\
$\rho_{S}$ & $:$ Particle density & {$\left[\mathrm{kg} / \mathrm{m}^{3}\right]$} \\
$\sigma_{r}$ & $:$ Principal radial stress & {$[\mathrm{Pa}]$} \\
$\sigma_{x}$ & $:$ Principal stress in flow direction & {$[\mathrm{Pa}]$} \\
$\tau_{w}$ & $:$ Shear stress at the wall & {$[\mathrm{Pa}]$} \\
$\phi$ & $:$ Angle of internal friction & {$[\mathrm{deg}]$} \\
$\phi_{s}$ & $:$ Ratio of straight length on periphery & {$[-]$} \\
\hline & a &
\end{tabular}

\section{Derivation of equation}

Figure 1 shows a stable transportation plug in a horizontal pipe. In a stable state, each plug sweeps up the stationary particles in front and leaves them behind a stationary layer of the same thickness ${ }^{(1)(7)}$. The plug length over which particles are filled in the cross-section is represented by $l_{P}$. The part in $l_{P}$ is called a plug, and the part which exists between a plug and a stationary bed is called the external plug. $l_{e x}$ is the length that is equal to the particle volume of the extra part divided by the cross-sectional area of the pipe. It is assumed that particles in $l_{P}$ are transported in a fixed state with each other, that is, they are transported as a lump, and $u_{s}$ is assumed to be the same regardless of the position in the plug. In addition, it is assumed that particles in an external plug are accelerated uniformly from 0 to $u_{s}$, and the mean particle velocity in an external plug is regarded as $0.5 u_{s}$.

The minute part length $d x$ within $l_{P}$ is taken, and the force balance is derived under the assumption that all stresses are function of $x$ only.

$$
\frac{d P}{d x}+\frac{d \sigma_{x}}{d x}+\frac{4 \tau_{w}}{D}=0
$$

Here, $\sigma_{x}$ is the principal internal particle stress in the flow direction, $P$ is the air pressure, $\tau_{w}$ 


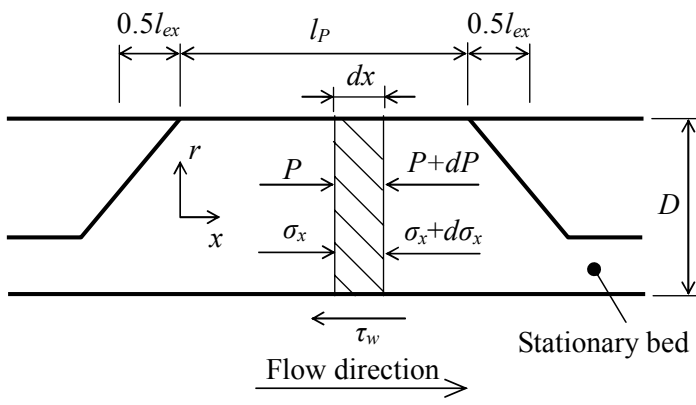

Fig.1 Pressure within a plug in horizontal pipe

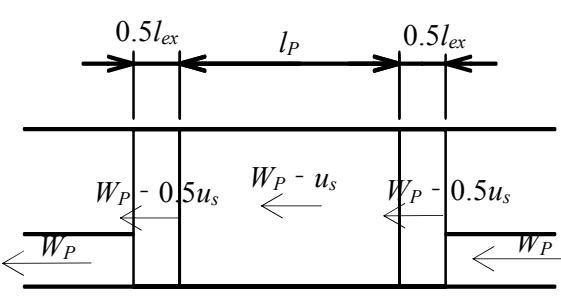

Fig.2 Particle velocity (Plug velocity axis)

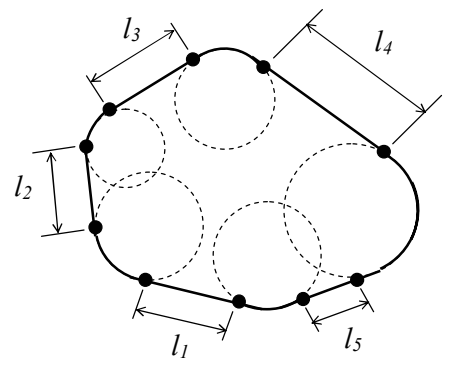

Fig.3 Ratio of straight length

is the shear stress at the wall, and $D$ is the pipe diameter. Konrad ${ }^{(1)}$ derived the equation for the principal internal particle stress in the radial direction $\sigma_{r}$ by deriving the Mohr's stress circle. For passive failure, i.e., $\sigma_{r}>\sigma_{x}$

$$
\begin{aligned}
& \sigma_{r}=K_{W} \sigma_{x}+\left(K_{W}+1\right) c \cos \phi \cos \left(\omega+\phi_{w}\right) \\
& K_{W}=\frac{1+\sin \phi \cos \left(\omega+\phi_{w}\right)}{1-\sin \phi \cos \left(\omega+\phi_{w}\right)} \\
& \sin \omega=\frac{\sin \phi_{w}}{\sin \phi} \\
& \tan \phi_{w}=\mu_{w}
\end{aligned}
$$

where $\phi$ is the angle of internal friction, and $c$ is the internal particle cohesion.

Next, the stress due to gravity on the pipe wall $P_{g}$ is considered. Konrad ${ }^{(1)}$, Pan and Wypych $^{(2)}$, and Tsuji ${ }^{(3)}$ obtained this stress from hydrostatic pressure theory. However, the particles are solid, so this method may not be applied. Instead, this paper considers $P_{g}$ as a unit force by plug weight.

$$
P_{g}=\frac{\frac{1}{4} \rho_{B} \pi D^{2} g d x}{\pi D d x}=\frac{1}{4} \rho_{B} g D
$$

Particles obey passive failure when they are transported near a rigid body ${ }^{(1)}$. Then, Eq.(7) can be derived from Eqs.(2) and (6).

$$
\sigma_{r}=K_{W} \sigma_{x}+\left(K_{W}+1\right) c \cos \phi \cos \left(\omega+\phi_{w}\right)+\frac{1}{4} \rho_{B} g D
$$

The pressure drop within $l_{P}$ is assumed constant in a stable plug transportation, and Eq.(8) is obtained by integrating for $\sigma_{x}$ from Eqs.(1), (7) and Coulomb's law.

$$
\sigma_{x}=k \exp \left[-\frac{4 \mu_{w} K_{W}}{D} x\right]-\frac{\Delta P}{l_{P}} \frac{D}{4 \mu_{w} K_{W}}-\frac{\left(K_{W}+1\right) c \cos \phi \cos \left(\omega+\phi_{w}\right)}{K_{W}}-\frac{\rho_{B} g D}{4 K_{W}}-\frac{C_{w}}{\mu_{w} K_{W}}
$$

In this equation, $k$ is a constant of integration, $\Delta P$ is the pressure drop through the plug, $\mu_{w}$ is the coefficient of friction and $C_{w}$ is the particle-wall cohesion. From Janssen's analysis $^{(1)(8)}$, stresses at the front and back end of a plug can be assumed to be the same value. Therefore, $\sigma_{x}=F$ at $x=0, l_{P}$.

$$
\frac{\Delta P}{l_{P}}=\frac{4 \mu_{w} K_{W} F}{D}+\frac{4 \mu_{w}\left(K_{W}+1\right) c \cos \phi \cos \left(\phi_{w}+\omega\right)}{D}+\rho_{B} g \mu_{w}+\frac{4 C_{w}}{D}
$$

Konrad et al. determined $F$ from the difference in the momentum between the stationary 
layer and $l_{P}$. However, his approach might not be applicable since there is $l_{e x}$ between them. Therefore, the axis of plug velocity $W_{P}$ is taken as shown in Fig.2, and $F$ is obtained by considering the momentum balance between $l_{e x}$ and $l_{P}$.

$$
F=\frac{1}{2} \frac{G_{s}}{A} u_{s}
$$

$G_{s}$ is the mass flow rate of particles that are taken into a plug and discharged from a plug. $u_{s}$ is different from $W_{P}$ due to the particle flow. As for $G_{s}$, Eq.(11) is obtained from Fig.2.

$$
G_{s}=A \rho_{B}\left(W_{p}-u_{s}\right)
$$

The analogy to gas-liquid systems is well-established for gas-fluidized beds by Brooke Benjamin $^{(9)}$. This is applied to the relation between $W_{P}$ and $u_{s}$.

$$
W_{p}=u_{s}+0.542 \sqrt{g D}
$$

Equation(13) is obtained from Eqs.(10) (12).

$$
F=0.271 \rho_{B} u_{S} \sqrt{g D}
$$

For non-cohesive particles,

$$
C_{w}=c=0
$$

Therefore, Eq.(15) is derived from Eqs.(9), (13) and (14).

$$
\frac{\Delta P}{l_{P}}=\mu_{w} \rho_{B} g+1.084 \mu_{w} K_{W} \rho_{B} u_{s} \sqrt{\frac{g}{D}}
$$

On the other hand, Ergun derived the equation for a pressure drop in a plug ${ }^{(10)}$. However, it is well known that there is an difference between calculations by Ergun's equation and experimental results due to $D$ and the particle shape ${ }^{(11)(12)}$. Therefore, the author suggests a modified Ergun's equation into which these factors are incorporated ${ }^{(6)}$.

$$
\frac{\Delta P}{l_{P}}=150\left(1+\frac{2 d_{P}\left(1-0.645 \phi_{s}\right)}{3 D(1-\varepsilon)}\right)^{2} \frac{\eta(1-\varepsilon)^{2}}{\varepsilon^{3}\left\{\left(1-0.645 \phi_{s}\right) d_{P}\right\}^{2}} U_{S P}+1.75 \frac{\rho_{a}(1-\varepsilon)}{\varepsilon^{3}\left(1-0.645 \phi_{s}\right) d_{P}} U_{S P}{ }^{2}
$$

Here, $\eta$ is the air viscosity, $d_{P}$ is the particle diameter, $\rho_{a}$ is the air density, $\varepsilon$ is the porosity $\left(=1-\rho_{B} / \rho_{S}\right), U_{S P}$ is the slip velocity and $\phi_{S}$ is the particle straight ratio. $\phi_{S}$ is the ratio of straight length $l_{i}$ to circumference $l_{s}$ in the second dimensional projection of the particle, as shown in Fig.3. The number of the linear portion is given as $N_{s}$, and $\phi_{s}$ is defined as follows.

$$
\phi_{s}=\sum_{i=1}^{N_{s}} l_{i} / l_{s}
$$

In Eq.(17), $\phi_{s}$ is a real number from 0 to 1 . For example, $\phi_{s}=0$ indicates a spherical particle and $\phi_{s}=1$ indicates a cubic particle. Then, $U_{S P}$ is treated as Eq.(18) in this paper.

$$
U_{S P}=U_{a}-u_{s}
$$

Here, $U_{a}$ is the air velocity. Equation(19) is obtained by solving for $u_{s}$ from Eqs.(15), (16) and (18).

$$
\begin{aligned}
& u_{s}^{2}-\left(\frac{a}{b \rho_{a}}+2 U_{a}+\frac{1.084 \mu_{w} K_{W} \rho_{B}}{b \rho_{a}} \sqrt{\frac{g}{D}}\right) u_{s}+\frac{a U_{a}}{b \rho_{a}}+U_{a}^{2}-\frac{\rho_{B} g \mu_{w}}{b \rho_{a}}=0 \\
& a=150\left(1+\frac{2 d_{P}\left(1-0.645 \phi_{s}\right)}{3 D(1-\varepsilon)}\right)^{2} \frac{\eta(1-\varepsilon)^{2}}{\varepsilon^{3}\left\{\left(1-0.645 \phi_{s}\right) d_{P}\right\}^{2}} \\
& b=1.75 \frac{(1-\varepsilon)}{\varepsilon^{3}\left(1-0.645 \phi_{s}\right) d_{P}}
\end{aligned}
$$

Two solutions are found for $u_{s}$ from Eq.(19), but the larger value gives a negative slip velocity. Therefore the smaller value is the true value for $u_{s}$. In short, $u_{s}$ is given by Eq.(22). $u_{s}=\frac{1}{2}\left(\frac{a}{b \rho_{a}}+2 U_{a}+\frac{1.084 \mu_{w} K_{W} \rho_{B}}{b \rho_{a}} \sqrt{\frac{g}{D}}\right)-\frac{1}{2} \sqrt{\left(\frac{a}{b \rho_{a}}+2 U_{a}+\frac{1.084 \mu_{w} K_{W} \rho_{B}}{b \rho_{a}} \sqrt{\frac{g}{D}}\right)^{2}-4 \times\left(\frac{a U_{a}}{b \rho_{a}}+U_{a}{ }^{2}-\frac{\rho_{B} g \mu_{w}}{b \rho_{a}}\right)}$

The pressure drop in a plug is obtained by substituting $u_{s}$ calculated from Eq.(22) into Eq.(15). However, Eq.(15) must be expanded for the total pipe length because there are not 
only plugs that are a particle lump, but also air lumps in the horizontal pipe. The relationship between the pipe length $L_{T}$ and $L_{P}$ is derived as

$$
L_{P}=\frac{M_{s} L_{T}}{A \rho_{B} u_{s}}
$$

The pressure drop through an air lump can be neglected because it is much smaller than that in the plug. So, substituting Eq.(23) for Eq.(15) can be regarded as an expansion for the pipe length.

$$
\frac{\Delta P}{L_{T}}=\frac{M_{s}}{A \rho_{B} u_{s}}\left[\rho_{B} g \mu_{w}+1.084 \mu_{w} K_{W} \rho_{B} u_{s} \sqrt{\frac{g}{D}}\right]
$$

Therefore, particle velocity and pressure drop in a horizontal pipe can be calculated from Eqs.(22) and (24).

\section{Experimental apparatus and method}

Figure 4 shows the experimental apparatus used for this study. Three pipe diameters were used, i.e., $D=25.6,38.0$ and $50.0 \mathrm{~mm}$. The total pipeline length is about $13 \mathrm{~m}$, and the pipe material is polyacrylic. An air chamber is used to collect the compressed air in an air source with an air compressor and a pressure-reducing valve is installed after the air chamber to regulate the compressed air. A flow meter is installed at the rear of the pressure reducing valve to measure the air flow rate $Q_{a}$ and a load cell in a blow tank is installed to measure the particle mass flow rate $M_{s}$. In addition, two pressure sensors are installed in the horizontal pipe to measure the pressure in the pipe, as shown in Fig.4. Pressure in the pipe is obtained as the mean value for $30 \mathrm{sec}$ captured every $0.05 \mathrm{sec}$ in stability plug transportation. The transportation conditions are set to become stable by a pressure reducing valve and a particle feeder valve. The transportation conditions are $U_{a}=1.1 \sim 5.0 \mathrm{~m} / \mathrm{s}$ and solid-air mass flow rate ratio $m_{T}=21 \sim 105$.

A high-speed camera and PIV are used to measure the particle velocity alteration from the stationary bed to $l_{P}$, and the distribution of the particle velocity in a plug in the horizontal and vertical directions. The validity of supposition in the derivation of the proposed equation is then verified. The frame rate of the high-speed camera is $2000 \mathrm{fps}$ and the resolution is $1024 \times 1024$ pixels. The interrogation window of the PIV is $45 \times 45$ pixels, and the correlation coefficient is more than 0.7 and the resolution of the PIV is 0.056 $\mathrm{mm} /$ pixel. A metal halide light is irradiated at the pipe wall and luminance distribution by

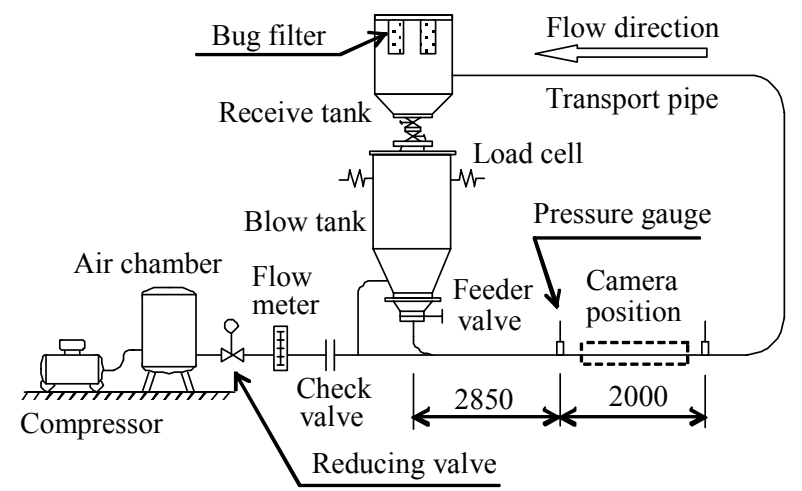

Fig.4 Experimental apparatus

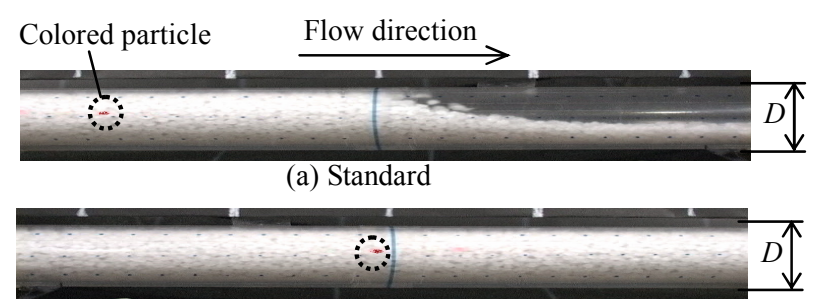

(b) After some frames

Fig.5 Measurement of particle velocity by digital video camera 
Table1 Particle properties

\begin{tabular}{|c|c|c|c|c|c|}
\hline Solids & Rapseed & $\begin{array}{c}\text { Polystyrol } \\
\text { pellet }\end{array}$ & $\begin{array}{c}\text { Polyethy } \\
\text {-lene pellet }\end{array}$ & $\begin{array}{c}\text { Nipolon } \\
\text { hard }\end{array}$ & $\begin{array}{c}\text { Plastic } \\
\text { pellet }\end{array}$ \\
\hline $\begin{array}{c}\text { Mean particle } \\
\text { diameter } \\
d_{P}(\mathrm{~mm})\end{array}$ & 1.98 & 2.80 & 3.27 & 3.54 & 6.00 \\
\hline $\begin{array}{c}\text { Straight } \\
\text { length ratio } \\
\phi_{s}\end{array}$ & 0.020 & 0.369 & 0.184 & 0.132 & 0.000 \\
\hline $\begin{array}{c}\text { Voidage } \\
\varepsilon \\
\end{array}$ & 0.420 & 0.383 & 0.378 & 0.416 & 0.461 \\
\hline $\begin{array}{c}\text { Bulk density } \\
\rho_{B}\left(\mathrm{~kg} / \mathrm{m}^{3}\right) \\
\end{array}$ & 435 & 678 & 591 & 531 & 572 \\
\hline $\begin{array}{c}\text { Particle density } \\
\rho_{S}\left(\mathrm{~kg} / \mathrm{m}^{3}\right)\end{array}$ & 1050 & 1100 & 950 & 910 & 1061 \\
\hline $\begin{array}{c}\text { Angle of } \\
\text { internal friction } \\
\phi(\operatorname{deg})\end{array}$ & 25.3 & 35.4 & 27.0 & 30.7 & 30.0 \\
\hline $\begin{array}{c}\text { Coefficient of } \\
\text { friction } \\
\mu_{w}{ }^{*} \\
\end{array}$ & 0.365 & 0.439 & 0.360 & 0.288 & 0.250 \\
\hline Shape & Sphere & Column & Disk & Oval & Sphere \\
\hline
\end{tabular}

particles in the plug is obtained. Therefore particle velocity is obtained by PIV. It is believed that the rotation does not affect the results of the PIV because there are no rolling particles in the plug. Though there are many researches which measured $W_{P}$, the result which measured particle velocity distribution in the plug has never been reported. In addition, a digital video camera is used to confirm the validity of the PIV results and to measure $u_{s}$. This camera's frame rate is $30 \mathrm{fps}$. Some particles are colored to facilitate the measurement of $u_{s}$. To measure $u_{s}$, the colored particles in the plug are first found in the image recorded by the digital video camera. Then, some frames are advanced and $u_{s}$ is calculated from the number of advanced frames and the particle transfer length, as shown in Fig.5. The high-speed camera and digital video camera are positioned within the dashed box in Fig.4. The high-speed camera is used for three different test conditions, i.e., $D=38$ and $50 \mathrm{~mm}$ polyethylene pellet, and $D=38 \mathrm{~mm}$ plastic pellet.

Rapeseed, polystyrol pellets, polyethylene pellets, nipolon hard and plastic pellets are used as the particles in the experiments. Table 1 shows their properties.

\section{Experimental result}

Figure 6 shows a result of the particle velocity vectors in a plug analyzed by PIV, which is the result of a plastic pellet and $m_{T}=62.8$. The vectors are the mean value for 50 frames. Figure 6 shows that all of the speed vectors point toward the flow direction, and the particles do not move in vertical direction.

Then, $u_{s}$ in flow direction measured by PIV is compared with that measured by the digital video camera in order to confirm the validity of the PIV result. $u_{s}$ by the digital video camera is the mean value of a hundred particles. Particle velocity by PIV is the mean value for 50 frames and three plugs in the area of $D \times D$. Figure 7 shows that each value almost agrees. Additionally, there were little error vectors in the PIV analysis and therefore results of the PIV are correct.

To clarify the particle velocity distribution in the plug, the relationship between the particle velocity by PIV and the position in the vertical direction $y / D$ is presented in Fig.8. Figure 8 shows that the velocity vectors of all positions are almost the same value regardless of the cross-sectional position. These results are the values at the wall. Tsuji ${ }^{(13)}$ and Chen ${ }^{(14)}$ suspected that there may be non-uniform velocity distribution in a plug. But in 


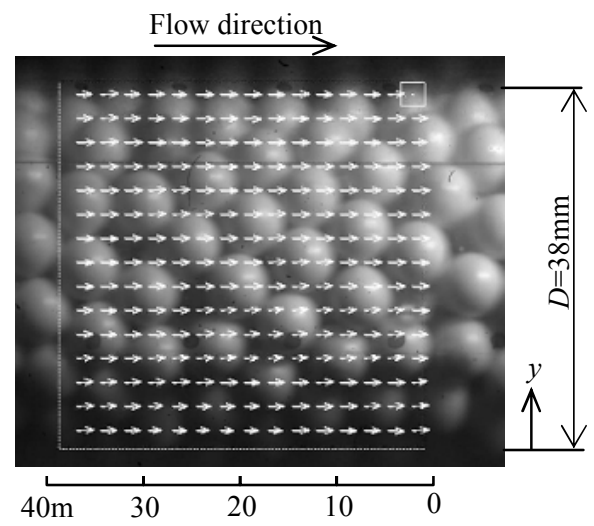

Fig.6 Velocity vector in a plug (Plastic pellet, $m_{T}=62.8$ )

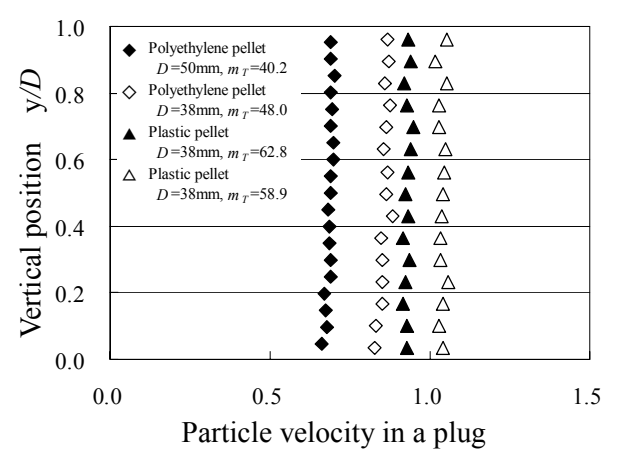

Fig.8 Particle velocity distribution in a plug

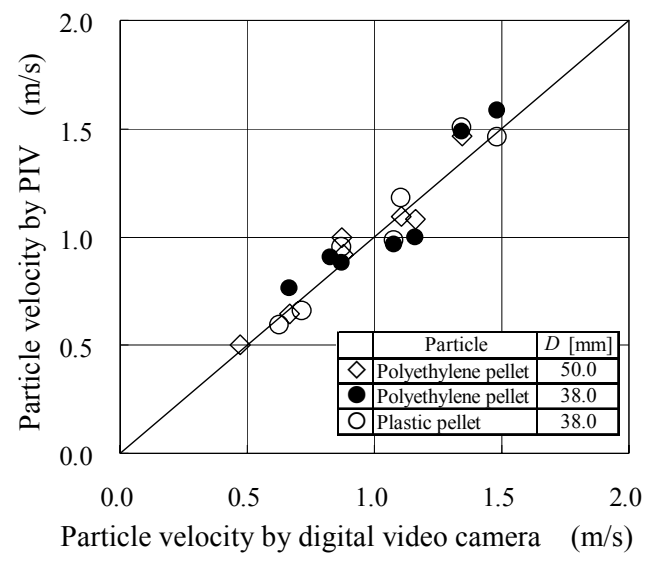

Fig.7 Confirmation on validity of analysis by PIV

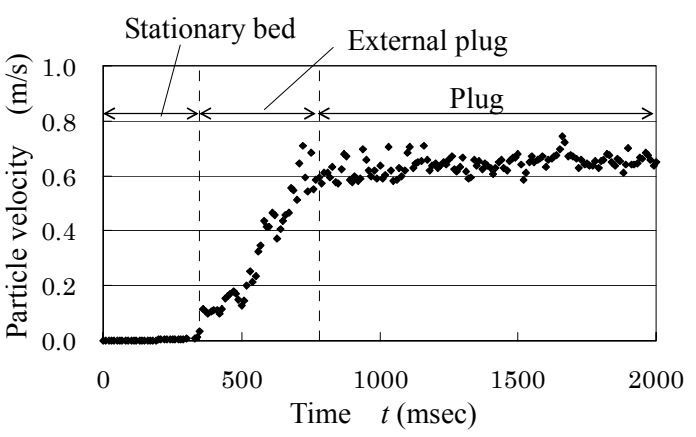

Fig.9 Particle velocity at each position (Polyethylene pellet, $D=50 \mathrm{~mm}, m_{T}=40.2$ )

the present study particle velocity is the same value in a cross-sectional area in the plug because there is uniform velocity distribution in Fig. 8 and in Tanaka's simulation research result ${ }^{(15)}$, and there are no rolling particles.

Next, the particle velocity variation in the mainstream direction from the stationary layer to $l_{P}$ is examined. The area at the same position for the mainstream direction is taken, and the velocities in each region of this area are measured by PIV and are shown in Fig.9. This particle velocity is the mean value over this area where particles had been satisfied to the pipe wall in order to remove float particles. Figure 9 shows there is no change in the particle velocity in the flow direction in the plug. From the result of Figs.6, 8 and 9, it is said that particles in $l_{P}$ are transported in a fixed state relative to each other, that is, they are transported in a lump. Therefore $u_{s}$ can be assumed to be a constant value regardless of the position in a plug. Additionally, Fig.9 shows particles in an external plug are accelerated uniformly, hence the mean particle velocity in an external plug can be regarded as $0.5 u_{s}$.

\section{Analysis of equation}

The validity of the equation for predicting particle velocity in a plug and pressure drop derived by Konrad ${ }^{(1)}$, Pan and $\mathrm{Wypych}^{(2)}$, Tsuji ${ }^{(3)}$, Mi and $\mathrm{Wypych}^{(4)}$ and $\mathrm{Aziz}^{(5)}$ is examined. Figures 10 to 15 show $U_{a}$ along the horizontal axis and the ratio of the predicted value to the measured one along the vertical axis. The ratio with respect to $u_{s}$ is shown as $\alpha$, and the ratio with respect to $\Delta P / L_{T}$ is shown as $\beta . u_{s}$ is taken for the mean value measured by the digital video camera since $u_{s}$ does not change in a plug. $u_{s}$ calculated from each researcher's equation is used when calculating $\Delta P / L_{T}$. Experiments on $u_{S}$ are not compared with the calculated value of Aziz at this time since he did not derive an equation on it. Pan and Wypych, and Mi and Wypych derived the equation about $W_{P}$, not $u_{s}$. Therefore, $u_{s}$ is calculated from Eq.(12) and compared with the experiments. Still, $\rho_{a}$, which is required to calculate these values, is obtained from the measured internal pipe pressure. 


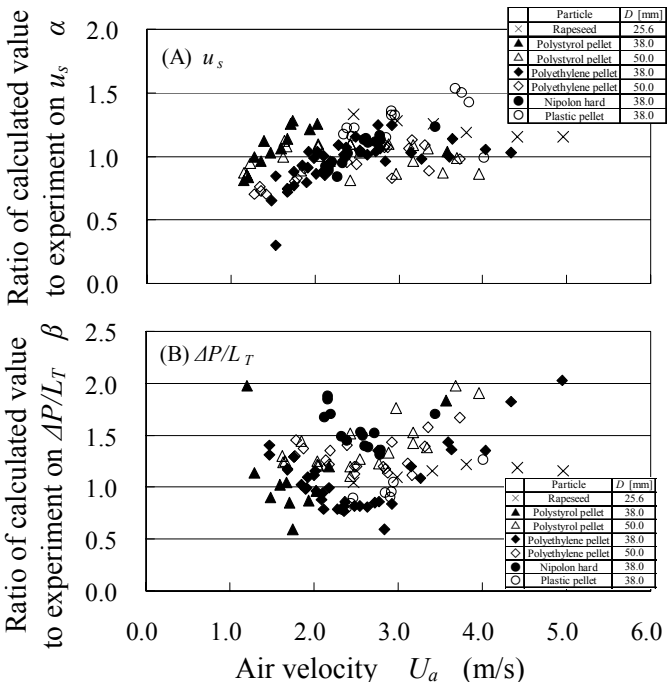

Fig.10 Comparison of experiment with calculation (Konrad)
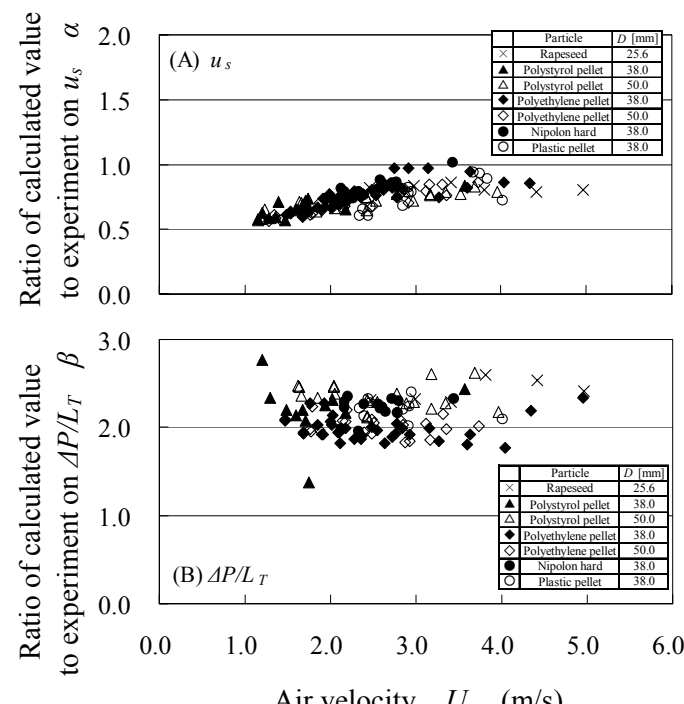

Fig.12 Comparison of experiment with calculation (Tsuji)

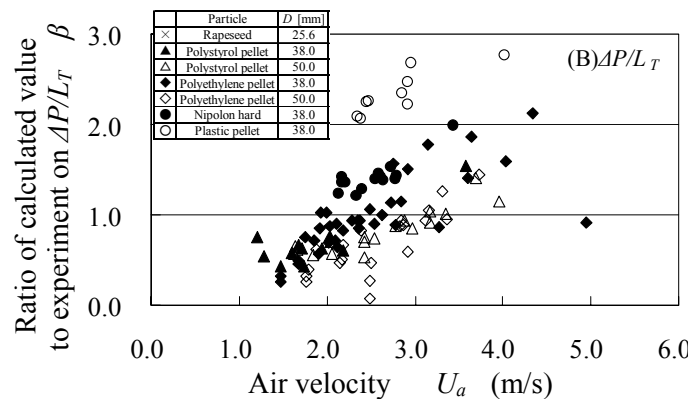

Fig.14 Comparison of experiment with calculation (Aziz)

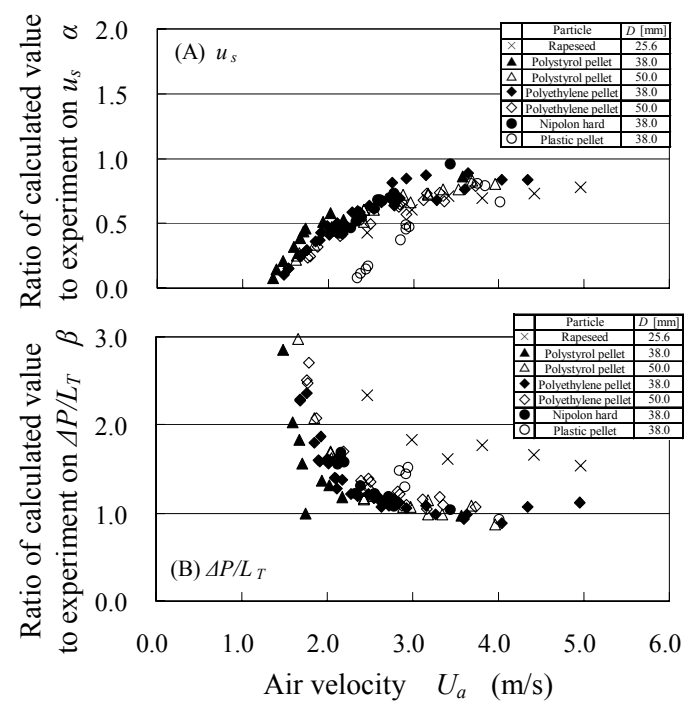

Fig.11 Comparison of experiment with calculation (Pan and Wypych)
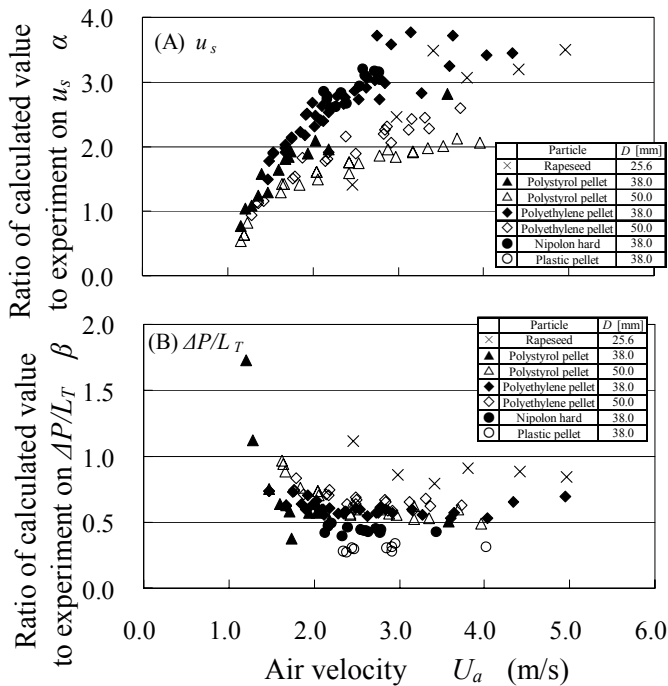

Fig.13 Comparison of experiment with calculation (Mi and Wypych)

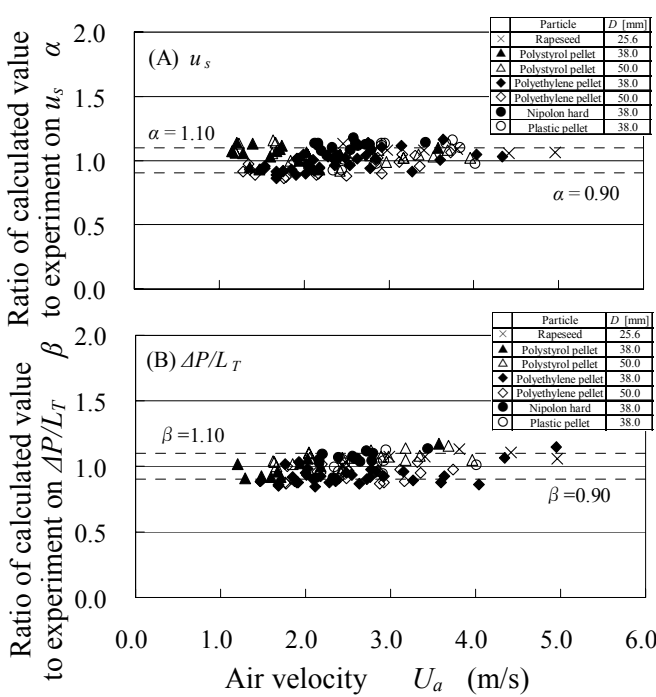

Fig. 15 Comparison of experiment with calculation (This study) 
Table2 Difference of pressure drop equation

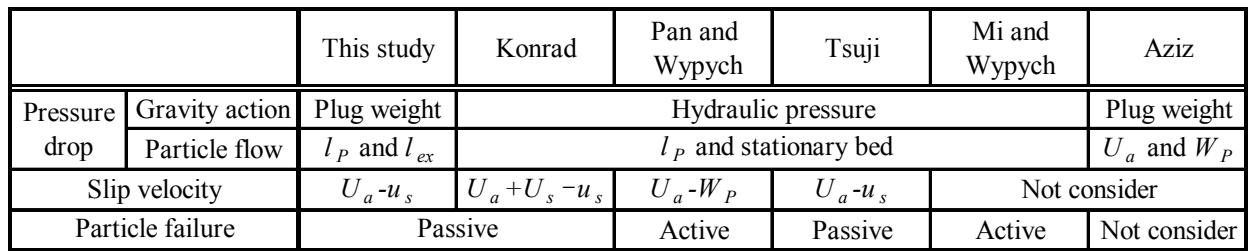

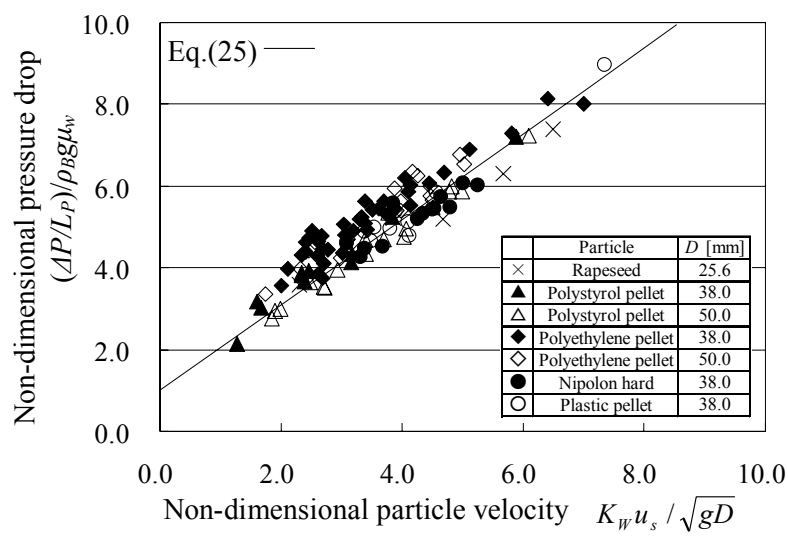

Fig.16 Pressure drop versus particle velocity

Table2 shows the difference of the pressure drop equation. The equations of Konrad, Pan and Wypych, Tsuji, Mi and Wypych, and Aziz are different from this study in terms of what gravity action is applied by the hydrostatic pressure. Additionally, when $F$ is introduced, Aziz obtained it from the difference between $U_{a}$ and $W_{P}$, while others obtained it from a momentum balance between the plug and stationary bed. Also, Konrad applied superficial particle velocity $U_{s}$ to slip velocity, and Pan and Wypych considered particles as active failure and the slip velocity as the difference between $U_{a}$ and $W_{P}$.

Figure 10 shows that the experimental results are different from the values predicted by Konrad because $\alpha$ ranges from 0.6 to 1.5 . Note that calculation conditions are different from Ergun's equation because the superficial particle velocity was applied to $U_{S P}$. Subsequent calculations agree with the experiments when the particles are polyethlene pellets at $D=38$ $\mathrm{mm}$, plastic pellets and rapeseed, and the $U_{a}$ is from 2.0 to $3.0 \mathrm{~m} / \mathrm{s}$. However, the calculations differ from the experimental results under other conditions. $\beta$ is larger than 1.0. This means that the pressure drop equation by Konrad estimates a value larger than actual value, and is applicable under limited conditions.

Figure 11 shows that the experimental results are different from the value predicted by Pan and Wypych. When $U_{a}$ is less than $3.0 \mathrm{~m} / \mathrm{s}, \alpha$ and $\beta$ are much different from 1.0, although the calculations have a good agreement with the experimental results when $U_{a}$ is larger than $3.0 \mathrm{~m} / \mathrm{s}$, except for rapeseed. This difference is possibly due to taking $U_{S P}$ from the difference between $U_{a}$ and $W_{P} . U_{a}$ becomes large, $u_{s}$ and $W_{P}$ also become larger. Therefore the effect of $0.542 \sqrt{g D}$ in Eq.(12) on $W_{P}$ or $U_{a}$ becomes smaller. But as $U_{a}$ becomes smaller, the effect of $0.542 \sqrt{g D}$ becomes larger. This could be an origin of the difference. Therefore, $U_{S P}$ cannot be taken for $U_{a}-W_{P}$.

$\alpha$ is smaller than 1.0 and $\beta$ is about 2.0 according to Tsuji's equation, as shown in Fig.12. $\alpha$ and $\beta$ are not shown in accordance to the experiments. The estimation of the effect of gravity action and particle flow on the pressure drop are far higher than the actual value.

$\alpha$ and $\beta$ calculated from the equation of Mi and Wypych completely deviate from the experimental values, as shown in Fig.13. Mi and Wypych deduced a relational expression on $u_{s}$ with $U_{a}$ from the experimental value under a specific condition. Therefore, the difference occurs because the applicability of the specific case is lacking.

Regarding the value of $\beta$ calculated from the Aziz equation in Fig.14, it is proven that 
there is completely no coincidence between the calculated value and the experimental value. From this result, the pressure drop by the particle flow cannot be considered at the difference between $U_{a}$ and $W_{P}$.

The above results indicate that all of the previously-derived equations on $u_{s}$ and $\Delta P / L_{T}$ have a difference relative to the experimental results. Figure 15 , on the other hand, shows that $\alpha$ and $\beta$ are mostly in the range 0.90 to 1.10 regardless of pipe diameter, type of particle and transportation conditions. Therefore, the difference of the equations proposed in this study for $u_{s}$ and $\Delta P / L_{T}$ is within $\pm 10 \%$ for all test conditions. This means that the proposed equations have high adaptability for types of particles, pipe diameter and transport conditions. In this study, the definition of slip velocity, the determination of $F$ and the estimation of the effect of gravity action and particle flux on pressure drop are all made appropriately to construct the correct prediction.

Additionally, the relationship between non-dimensional particle velocity and non-dimensional pressure drop in a plug is shown in Fig.16 in order to confirm the validity of the estimation by the proposed equation for pressure drop due to gravity action and particle flow. The plotting point in the figure is the value that is measured $\triangle P$ and divided by $L_{P}$ calculated from Eq.(23) and $\rho_{B} g \mu_{w}$. Then, the experimental value of $u_{s}$ was used in Eq.(23). The continuous line in the figure is the prediction line from Eq.(25), which is derived from Eq.(24), divided by Eq.(23) and $\rho_{B} g \mu_{w}$.

$$
\frac{\Delta P}{L_{P}} \cdot \frac{1}{\rho_{B} g \mu_{w}}=1+1.084 \frac{K_{W} u_{s}}{\sqrt{g D}}
$$

The first term on the right side of Eq.(25) represents the pressure drop due to gravity, and the second term represents the pressure drop due to the particle flow. Therefore, the vertical axis at $u_{s}=0$ in Fig. 16 indicates the pressure drop due to gravity and the slope of the line indicates the pressure drop due to the particle flow. Fig.16 shows that the experimental results agree with the prediction equation of this study. Therefore, it is said that the prediction equation of this study precisely estimates pressure drop by gravity and particle flow in all test conditions.

\section{Conclusion}

In this paper, particle flow in the plug and transportation characteristic are analyzed in granular particle plug transportation through the horizontal pipe. As particles, rapeseed, polystyrol pellets, polyethylene pellets, nipolon hard and plastic pellets are used. Pipe diameters are 25.6, 38.0 and $50.0 \mathrm{~mm}$ and length is about $13 \mathrm{~m}$. Transportation conditions are $U_{a}=1.1 \sim 5.0 \mathrm{~m} / \mathrm{s}$ and $m_{T}=21 \sim 105$. The results are as follows:

1) Equations on particle velocity in the plug and pressure drop applied modified Ergun's equation have been formulated. Calculations by these equations agree with experiments within $10 \%$ in all test condition. Additionally, the pressure drop estimations due to gravity action and particle flow are appropriate.

2) Particle velocity at the wall vicinity is constant regardless of the position from the result of high speed camera and PIV. Then it is said particles in the plug are transported in a fixed state with each other from this study's and Tanaka's research result.

3) Particles in an external plug are accelerated uniformly and they all move with the same velocity in the plug from the result of high speed camera and PIV.

\section{Appendix}

Each researcher's equation is shown in the following. Eq.(14) is applied during calculation in this paper.

(1) Konrad

$$
\frac{\Delta P}{L_{T}}=\frac{M_{s}}{A \rho_{B} u_{s m}}\left[2 g \mu_{w} \rho_{B}+2.168 \mu_{w} K_{W} \rho_{B} u_{s m} \sqrt{\frac{g}{D}} \cdot \frac{1-2 M_{s} / \rho_{B} A u_{s m}}{1-M_{s} / \rho_{B} A u_{s m}}\right]
$$




$$
\begin{aligned}
& u_{s m}{ }^{3}+u_{s m}{ }^{2}\left(-\frac{\left(a^{\prime}+f\right)}{b^{\prime} \rho_{a}}-2\left(U_{a}+U_{s}\right)-\frac{M_{s}}{\rho_{B} A}\right)+u_{s m}\left(\frac{a^{\prime}\left(U_{a}+U_{s}\right)}{b^{\prime} \rho_{a}}+\left(U_{a}+U_{s}\right)^{2}-\frac{2 g \rho_{B} \mu_{w}}{b^{\prime} \rho_{a}}\right. \\
& \left.+\frac{M_{s}\left(a^{\prime}+2 f\right)}{\rho_{B} A b^{\prime} \rho_{a}}+\frac{2 M_{s}}{\rho_{B} A}\left(U_{a}+U_{s}\right)\right)-\frac{M_{s}}{\rho_{B} A}\left(\frac{a^{\prime}\left(U_{a}+U_{s}\right)}{b^{\prime} \rho_{a}}+\left(U_{a}+U_{s}\right)^{2}-\frac{2 g \rho_{B} \mu_{w}}{b^{\prime} \rho_{a}}\right)=0 \\
& a^{\prime}=150 \frac{\eta(1-\varepsilon)^{2}}{\varepsilon^{3} d_{P}^{2}} \\
& b^{\prime}=1.75 \frac{(1-\varepsilon)}{\varepsilon^{3} d_{P}} \\
& f=2.168 \mu_{w} K_{W} \rho_{B} \sqrt{g / D} \\
& U_{s}=M_{s} / \rho_{s} A \\
& u_{s m}=\frac{l_{P} u_{s}+\left(l_{e x} u_{s} / 2\right)}{l_{P}+l_{e x}}
\end{aligned}
$$

Usually, $l_{P}$ is far larger than $l_{e x}$. Therefore $u_{s m}$ is treated as $u_{s}$ in this paper.

(2) Pan and Wypych

$$
\begin{aligned}
& \frac{\Delta P}{L_{T}}=\frac{M_{s}}{A(1-\alpha) \rho_{B} W_{P}}\left[2.168 \mu_{w} \rho_{B} K_{W}^{\prime} \sqrt{\frac{g}{D}} W_{P}+\rho_{B} g \mu_{w}\left(2-1.175 K_{W}^{\prime}\right)\right] \\
& \alpha=0.542 \sqrt{g D} / W_{P} \\
& K_{W}{ }^{\prime}=\frac{1-\sin \phi \cos \left(\omega-\phi_{w}\right)}{1+\sin \phi \cos \left(\omega-\phi_{w}\right)} \\
& A_{1} W_{P}^{2}+A_{2} W_{P}+A_{3}=0 \\
& A_{1}=b^{\prime} \rho_{a} \\
& A_{2}=-\left[a^{\prime}+2.168 \mu_{w} K_{W} \rho_{B} \sqrt{\frac{g}{D}}+2 b^{\prime} \rho_{a} U_{a}\right] \\
& A_{3}=a^{\prime} U_{a}+b^{\prime} \rho_{a} U_{a}^{2}-\rho_{B} g\left(2-1.175 \mu_{w} K_{W}^{\prime}\right)
\end{aligned}
$$

(3) Tsuji

$$
\frac{\Delta P}{L_{T}}=\frac{M_{s}}{\frac{\pi}{4} D^{2} u_{s}(1-\varepsilon) \rho_{S}}\left[2.168 \mu_{w} K_{W} \rho_{B} u_{s} \sqrt{\frac{g}{D}}+2 \mu_{w} \rho_{B} g+\frac{4}{D}\left\{\mu_{w}\left(K_{W}+1\right) c \cos \phi\left(\omega+\phi_{w}\right)+C_{w}\right\}\right]
$$

$$
C_{1}\left(1-u_{s} / U_{a}\right)+C_{2}\left(1-u_{s} / U_{a}\right)^{2}=C_{3} u_{s} / U_{a}+C_{4}+C_{5}
$$

$C_{1}=150 \frac{(1-\varepsilon)^{2}}{\varepsilon^{3}} \frac{\rho_{a}}{\left(d_{P} / D\right)^{2} U_{a} D \eta}$

$C_{2}=1.75 \frac{1-\varepsilon}{\varepsilon^{3}} \frac{1}{d_{P} / D}$

$C_{3}=2.168 \mu_{w} K_{W}(1-\varepsilon) \frac{\rho_{S}}{\rho_{a} F_{r a}}$

$C_{4}=2 \mu_{w}(1-\varepsilon) \frac{\rho_{S}}{\rho_{a} F_{r a}{ }^{2}}$

$C_{5}=\frac{4}{\rho_{a} U_{a}^{2}}\left\{\mu_{w}\left(K_{W}+1\right) c \cos \phi\left(\omega+\phi_{w}\right)+C_{w}\right\}$

$F_{r a}=U_{a} / \sqrt{g D}$

(4) Mi and Wypych

$$
\begin{aligned}
& \frac{\Delta P}{L_{T}}=\left(1+1.084 K_{W}{ }^{\prime} F_{r s}^{0.5}+0.542 F_{r s}^{-0.5}\right) \frac{2 g \mu_{w} M_{s}}{A W_{P}} \\
& W_{P}=k\left(U_{a}-U_{a \min }\right) \\
& U_{a \min }=\frac{\rho_{S} g \tan \phi_{w} \varepsilon^{3} d_{P}^{2}}{180(1-\varepsilon) \eta} \\
& k=105 \frac{\varepsilon d_{P}}{D}\left(\frac{\tan \phi_{w}}{\tan \phi}\right)^{1 / 3} \\
& F_{r s}=W_{P}{ }^{2} / g D
\end{aligned}
$$


(5) Aziz

$$
\begin{aligned}
& \frac{\Delta P}{L_{P}}=E+\rho_{B} g K_{W}\left(\frac{U_{a}}{W_{P}}-1\right)\left(2 \tan \phi_{w}+\frac{4 \tan \phi}{\pi}\right) F_{r s} \\
& E=\frac{\Gamma}{2}+\frac{4 c}{\pi D} \pm \frac{4\left(K_{W}+1\right) c \cos \phi \tan \phi \cos (\omega \pm \phi)}{\pi D} \\
& \Gamma=\frac{4 C_{w}}{D} \pm \frac{4}{D}\left(K_{W}+1\right) c \cos \phi \tan \phi_{w} \cos \left(\omega+\phi_{w}\right)
\end{aligned}
$$

In this study, Eq.(23) is applied for Eq.(53) to obtain $\Delta P / L_{T}$.

$$
\frac{\Delta P}{L_{T}}=\frac{M_{s}}{A \rho_{B} u_{s}}\left[E+\rho_{B} g K_{W}\left(\frac{U_{a}}{W_{P}}-1\right)\left(2 \tan \phi_{w}+\frac{4 \tan \phi}{\pi}\right) F_{r s}\right]
$$

\section{References}

(1) Konrad, K., Prediction of the pressure drop for horizontal dense phase pneumatic conveying of particles, Proceedings of 5th conference BHRA Fluid engineering, Paper E1 (1986) , pp.225-244

(2) Pan, R. and Wypych, P.W., Pressure drop and slug velocity in low-velocity pneumatic conveying of bulk solids, Powder Technology, Vol.94 (1997), pp.123-132

(3) Tsuji,Y., Pneumatic transportation basic (in Japanese), Yokendo (1984), pp.114-136

(4) Mi, B. and Wypych, P.W., Pressure drop prediction in low-velocity pneumatic conveying, Powder Technology, Vol. 81 (1994), pp.125-137

(5) Aziz, A.B. and Klinzing, G.E., Dense phase plug flow transfer - The 1-inch horizontal flow-, Powder Technology, Vol. 62 (1990), pp.41-49

(6) Kofu, K., Ochi, M. and Takei, M., Proposal of Ergun's Equation Modified with Ratio of Straight Length to Particle Perimeter and Pipe Wall Effect, Journal of the Society of Powder Technology, Japan, Vol.44, No.4 (2007), pp.259-266

(7) Jaworski, A.J. and Dyakowski, T., Investigations of flow instabilities within the dense pneumatic conveying system, Powder Technology, Vol.125 (2002), pp.279-291

(8) Janssen, H.A., Versuche uber Getreidedruck in Silozellen, Z.ver.deut.Ing., 39, pp.1045 (1895 In German)

(9) Brooke Benjamin,T., Gravity currents and related phenomena, The Journal of Fluid Mechanics, Vol.31, Part2, section3 (1968), pp.209-248

(10) Ergun, S., Fluid flow through packed columns, Chemical Engineering Progress, Vol.48, No.2 (1952), pp.89-94

(11) Nemec, D. and Levec, J., Flow through packed reactors, Chemical Engineering Science, Vol.60 (2005), pp.6947-6957

(12) Yamazaki, R., Kozeny-Carman's Equation, Journal of the Society of Powder Technology, Japan, Vol.11, No.71 (1974), pp.428-43

(13) Tsuji, Y. and Morikawa, Y., Plug flow of coarse particles in a horizontal pipe, Journal of Fluids Engineering Transactions of the ASME, Vol.104 (1982), pp.198-206

(14) Chen, D., Klausner, J.F. and Mei, R., A fluid mechanics approach to describing the behavior of pneumatically conveyed powder plugs, Powder Technology, Vol.124 (2002), pp.127-137

(15) Tanaka, T., Ishida, T. and Tsuji, Y., Direct numerical simulation of granular plug flow in a horizontal pipe (the case of cohesionless particles), Transactions of the Japan Society of Mechanical Engineers, Series B, Vol.57, No.534 (1991), pp.456-463 